\title{
Laryngeal Mask Airway for Surfactant Administration in a Newborn Animal Model
}

\author{
KARI D. ROBERTS, ANDREA L. LAMPLAND, PATRICIA A. MEYERS, CATHY T. WORWA, BRENDA J. PLUMM,
} AND MARK C. MAMMEL

Department of Pediatrics [K.D.R., A.L.L., M.C.M.], University of Minnesota, Amplatz Children's Hospital, Minneapolis, Minnesota 55455; Infant Diagnostics and Research Center [K.D.R., A.L.L., P.A.M., C.T.W., B.J.P., M.C.M.], Children's Hospitals \& Clinics of Minnesota, St Paul, Minnesota 55404

\begin{abstract}
Premature infants are subjected to adverse effects of intubation to benefit from surfactant. We hypothesized that administration of surfactant through a laryngeal mask airway (LMA) is as effective as administration through an endotracheal tube (ETT) and that time and physiologic changes during instrumentation will be less in the LMA group. This study is a randomized, controlled trial using newborn pigs. Lung injury was induced via surfactant washout. Animals were randomized into groups: 1) LMA placed, no surfactant administered (control; $n=8$ ); 2) surfactant via an LMA (LMA group; $n=8$ ); and 3 ) surfactant via an ETT (ETT group; $n=8$ ). We demonstrated that partial pressure of oxygen in arterial blood $\left(\mathrm{PaO}_{2}\right)$ levels of the LMA and ETT groups were not statistically different. Time for successful placement of LMA was $19 \pm 1 \mathrm{~s}$ versus ETT $123 \pm 35 \mathrm{~s}$ (mean $\pm \mathrm{SEM}$ ); number of attempts for successful LMA placement was 1.1 (1-2) versus ETT 1.9 (1-7) [mean (range)]. Administration of surfactant via an LMA compared with an ETT resulted in similar improvements in oxygenation. Placement of the device required less time and fewer attempts. These data suggest that further study in human neonates is justified. If proven effective, some infants with respiratory distress may be able to receive surfactant while avoiding intubation. (Pediatr Res 68: 414-418, 2010)
\end{abstract}

$\mathrm{R}^{\mathrm{c}}$ espiratory distress syndrome (RDS) remains the single most important cause of morbidity and mortality in premature infants (1). Clinical studies have shown that surfactant therapy dramatically improves survival and respiratory outcomes of premature infants with RDS (2). Currently, intubation with an endotracheal tube (ETT) is required to deliver surfactant. Intubation, however, is an invasive procedure associated with adverse physiologic effects including bradycardia $(3,4)$, fluctuations in blood pressure (BP) (3-8), hypoxia $(3,4,7,9,10)$, and increases in intracranial pressure $(4-6,8,11)$.

The laryngeal mask airway (LMA) can be used to provide assisted respiratory support in infants, children, and adults without the need for direct visualization or passage of a device through the glottis (12). The LMA is a disposable, silicone rubber tube connected to an elliptical mask with a soft, inflatable cuff (Fig. 1). It is inserted orally using the operator's

Received January 5, 2010; accepted June 22, 2010.

Correspondence: Kari D. Roberts, M.D., Department of Pediatrics, University of Minnesota, Amplatz Children's Hospital, 420 Delaware St SE, MMC 39, Minneapolis, MN 55455; e-mail: rober694@umn.edu

Supported by grants from the Neonatal Resuscitation Program, the American Academy of Pediatrics and the Education \& Research Committee of Children's Hospitals and Clinics of Minnesota Foundation. Surfactant was provided by Discovery Laboratories, Inc. index finger and guided along the hard palate without laryngoscopy or other instruments. With cuff inflation, occlusion of the esophagus occurs and ventilation is directed down the trachea via openings in the mask lumen.

In the Neonatal Intensive Care Unit (NICU) setting, the LMA is most frequently used to establish an airway if intubation attempts have been unsuccessful. Although case reports have described successful use of an LMA for surfactant administration $(13,14)$, no systematic evaluation of its effectiveness has been performed. We designed a randomized, controlled study in newborn pigs to test the effectiveness of surfactant administered via an LMA compared with surfactant administered via an ETT. We hypothesized that improvements in oxygenation would be similar between the LMA and ETT groups suggesting that the LMA is as effective as an ETT for delivering surfactant to the lungs. In addition, we hypothesized that the time, number of attempts, and adverse physiologic changes during the placement of the LMA device would be less compared with placement of an ETT.

\section{METHODS}

Study design. This study was approved by the Institutional Animal Care and Use Committee of Children's Hospitals of Minnesota. Animal care was conducted in accordance with the National Institutes of Health guidelines (15). We created acute lung injury and surfactant deficiency in newborn pigs using surfactant washout with normal saline (16). The study was completed in two phases. Phase I was a proof of concept design to determine the feasibility of administering surfactant via an LMA device. Phase II consisted of two components. The first component compared time, number of attempts, and physiologic changes [heart rate (HR) and arterial oxygen saturation $\left(\mathrm{SaO}_{2}\right)$ ] during placement of the LMA or ETT performed by one of three research associates skilled in airway management of pigs. The second component was a randomized, controlled trial comparing the pulmonary efficacy [determined by partial pressure of oxygen in arterial blood $\left(\mathrm{PaO}_{2}\right)$ levels] of surfactant administered via an LMA compared with direct intratracheal instillation via an ETT. Randomization occurred by computer generated group assignments that were placed in sealed, opaque envelopes and opened in a consecutive manner.

Animal preparation. Newborn pigs (age 1-2 d old) were initially anesthetized with intramuscular ketamine $(50 \mathrm{mg} / \mathrm{kg} / \mathrm{dose})$. The internal carotid artery and external jugular vein were catheterized for monitoring blood gases, intravascular pressures, and administration of fluids and medications. Analgesia was maintained with continuous i.v. ketamine with a starting dose of 1 $\mathrm{mg} / \mathrm{kg} / \mathrm{h}$ and titrated by $1 \mathrm{mg} / \mathrm{kg} / \mathrm{h}$ to a maximum dose of $6 \mathrm{mg} / \mathrm{kg} / \mathrm{h}$ if the pig was agitated or if HR was consistently $>220$ beats per minute (bpm). If apnea

\footnotetext{
Abbreviations: ETT, endotracheal tube; HR, heart rate; LMA, laryngeal mask airway; nCPAP, nasal continuous positive airway pressure; $\mathbf{S a O}_{2}$, arterial oxygen saturation
} 


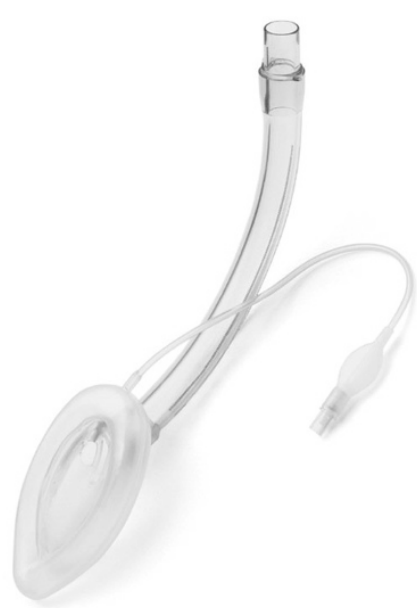

Figure 1. LMA. Image courtesy of LMA North America, Inc.

occurred, the ketamine drip was stopped for $15 \mathrm{~min}$ and restarted at half the previous dose.

Phase I: Feasibility study $(\mathbf{n}=4)$. Acute lung injury was induced by repeated normal saline lavage where aliquots of normal saline $(20 \mathrm{~mL} / \mathrm{kg})$ were instilled, allowed to dwell for $3 \mathrm{~min}$, and then suctioned. The process was repeated until $\mathrm{PaO}_{2}$ was $<150 \mathrm{~mm} \mathrm{Hg}$ on fraction of inspired oxygen $\left(\mathrm{FiO}_{2}\right)$ of 1.0. During the preparation period, animals were supported with mechanical ventilation (Dräger Babylog 8000, Dräger America, Telford, PA); ventilator settings were pressure support ventilation with volume guarantee (PSV $+\mathrm{VG})$, tidal volume (TV) of $8 \mathrm{~mL} / \mathrm{kg}$, and positive end-expiratory pressure (PEEP) of $6 \mathrm{~cm} \mathrm{H}_{2} \mathrm{O}$. Ventilator rate was adjusted to maintain partial pressure of carbon dioxide in the arterial blood $\left(\mathrm{PaCO}_{2}\right)$ between 40 and 50 $\mathrm{mm} \mathrm{Hg}$.

After lung injury was induced, animals were extubated and placed on nasal continuous positive airway pressure (nCPAP) of $6 \mathrm{~cm} \mathrm{H}_{2} 0$ using binasal, short prongs. Baseline vital signs [ $\mathrm{HR}$, respiratory rate $\left.(\mathrm{RR}), \mathrm{BP}, \mathrm{SaO}_{2}\right]$ and arterial blood gas (ABG) (via blood gas monitor, International Biomedical, Ltd, Austin, Tx) were obtained after 5 min of nCPAP. An LMA (LMA UniqueSize 1, The Laryngeal Mask Company Limited, San Diego, CA) was then placed and the cuff inflated with $3 \mathrm{~mL}$ of air. Surfactant $(5.8 \mathrm{~mL} / \mathrm{kg}$, Surfaxin, lucinactant, Discovery Laboratories, Inc., Warrington, PA) was administered in two aliquots as a bolus down the top of the LMA. Vital signs were continuously monitored (Space Labs, Redmond, WA). The following factors were assessed: 1) Did placing an LMA elicit negative side effects such as agitation (defined as excessive movement) or unfavorable changes in vital signs [defined as $\mathrm{HR}<100$, mean $\mathrm{BP}(\mathrm{MBP})<80 \%$ of baseline, or $\mathrm{SaO}_{2}$ $<75 \%]$ ? 2) Did surfactant administration via an LMA elicit negative side effects such as agitation or unfavorable changes in vital signs? 3) Did surfactant administration via an LMA elicit laryngospasm? 4) Did surfactant administered down an LMA leak around the inflatable cuff?

To access for leakage around the inflatable cuff, methylene blue was added to the surfactant. A flexible video-scope was placed in the posterior pharynx and surfactant administration was recorded and analyzed. At the completion of the study, the upper airway, lungs, and upper gastrointestinal system were dissected and analyzed to determine whether surfactant staining was present in these organs.

Phase II: $(n=30)$

Component 1: Placement of airway device. In an effort to minimize bias in the time and number of attempts data due to how "easy" or "difficult" an individual pig was to intubate, animals served as their own control by having the same research associate place an LMA before intubating with a 3.0 ETT for induction of acute lung injury. After being properly positioned, a pulse oximeter was placed and an $\mathrm{SaO}_{2} \geq 95 \%$ was confirmed. Placement of an LMA then commenced followed by placement of an ETT. For both devices, the placement attempt was terminated if the $\mathrm{SaO}_{2}$ fell below $75 \%$ or the attempt exceeded $30 \mathrm{~s}$. Blow by oxygen was administered and a repeat attempt was initiated once $\mathrm{SaO}_{2}$ was $\geq 95 \%$. Placement of the device was considered successful when there was confirmation of good breath sounds, vapor present in the device, and end-tidal $\mathrm{CO}_{2}$ detection.

A custom designed data acquisition system was used to simultaneously record video information and analog physiologic data. Digital video data obtained from a 3CCD digital video camera (Model TRV-950, Sony Electrics, Oradell, NJ) and analog signals from the oximeter (Radical, Masimo Corporation, Irvine, CA) were processed through a data acquisition board (Model PCI-6014, National Instruments, Austin, TX) and analyzed using a process-specific program that links them in time. The actual data sampling rate was $16 \mathrm{~Hz}$ per channel. Both the video signal and physiology data could be played back and viewed on the same screen, thereby allowing accurate identification of the initiation and completion of any intervention to the nearest second. $\mathrm{HR}$ and $\mathrm{SaO}_{2}$ were calculated and stored for subsequent analysis (ProFox software, Escondido, CA). Duration of hypoxia was determined at oxygen saturation thresholds of $\mathrm{SaO}_{2} \leq 85 \%, \leq 70 \%$, and $\leq 60 \%$ (levels chosen as thresholds felt to be clinically relevant). HR measurements were analyzed as the absolute change from baseline. Baseline values were obtained before placement of the airway device. Procedural values commenced with the placement of the device and ended once successful placement of the device was confirmed.

Videotape of the procedure was reviewed to determine time and number of attempts needed to successfully place the airway device. Duration of time for each attempt was defined as the duration from first insertion of the instrument (LMA or laryngoscope) until the operator's index finger or laryngoscope was removed from the mouth. Duration of time for successful placement of the airway device was the sum of the time required for each attempt. A placement attempt was defined as placement of the LMA or laryngoscope in the mouth, regardless of whether an attempt was made to inflate the LMA cuff or pass an ETT through the glottis.

Component 2: Randomized, controlled trial $(n=30)$. After lung injury was induced according to the method described in section Phase I: Feasibility Study, animals were extubated and placed on nCPAP of $6 \mathrm{~cm} \mathrm{H}_{2} 0$. Animals were randomized into one of the following groups:

1. LMA placed, mechanical ventilation $\times 5$ min through LMA, no surfactant administered, LMA removed and placed back on nCPAP (control group).

2. LMA placed, surfactant administered through the LMA, followed by $5 \mathrm{~min}$ of mechanical ventilation, then LMA removed and placed back on nCPAP (LMA group).

3. Intubated, surfactant administered through the ETT, followed by $5 \mathrm{~min}$ of mechanical ventilation, then ETT removed and placed back on nCPAP (ETT group).

Ventilator settings for all three groups were PSV + VG, TV $8 \mathrm{~mL} / \mathrm{kg}$, PEEP $6 \mathrm{~cm} \mathrm{H} \mathrm{H}_{2}$, rate $40 \mathrm{bpm}$, and $\mathrm{FiO}_{2}$ 1.0. ABGs and vital signs were continuously monitored. Physiologic data recording points were before lung injury, after lung injury but before nCPAP application, 5 min after nCPAP applied, $30 \mathrm{~min}$ after surfactant administration, and every hour during the $3.5 \mathrm{~h}$ study. Pigs breathed spontaneously throughout the study and were suspended using a sling in their normal upright posture. At the end of the study, the pigs were euthanized with an overdose of i.v. ketamine $(100 \mathrm{mg} / \mathrm{kg})$ and a bolus of i.v. $\mathrm{KCl}$ to induce cardiac arrest.

Sample size and statistics analysis. Sample size was determined from a previous study investigating lung injury in pigs treated with nCPAP versus mechanical ventilation (17). This previous study is similar to our study in that the pigs were of similar age and weight and lung injury was induced in the same manner. $\mathrm{PaO}_{2}$ levels before and after surfactant therapy in the previous study $\left(\mathrm{PaO}_{2} 80\right.$ torr presurfactant to 350 torr postsurfactant resulting in a change of 270 torr) were used to calculate sample size needed for our study. Using these data, a sample size of 21 pigs (7 per group) was required to show a change in $\mathrm{PaO}_{2}$ of 270 torr with power 0.8 and $\alpha<0.05$. To ensure an adequate number of subjects and account for dropouts because of technical malfunction, injury or death, 10 pigs were randomized to each group for a total of 30 pigs. The number of pigs used for the feasibility study $(n=4)$ was based on our previous experience with this animal model.

Statistical analysis was performed with GraphPad Prism (Version 5.0, GraphPad Software, Inc, LA Jolla, CA). Pulmonary efficacy of surfactant administration was determined by comparing $\mathrm{PaO}_{2}$ levels among groups. Vital signs and blood gas parameters thorough the duration of the study were compared using ANOVA with post hoc tests. Duration of time and number of attempts required to successfully place an LMA versus ETT and physiologic parameters during the placement procedure were compared using paired $t$ tests or $\chi^{2}$ testing as appropriate. A $p$-value of $<0.05$ was considered statistically significant.

\section{RESULTS}

Phase I: Feasibility study. Four pigs were used for the feasibility study. The animals tolerated the procedure well and did not exhibit excessive agitation or unfavorable changes in vital signs during placement of the LMA or surfactant administration. No pigs demonstrated laryngospasm. Review of 


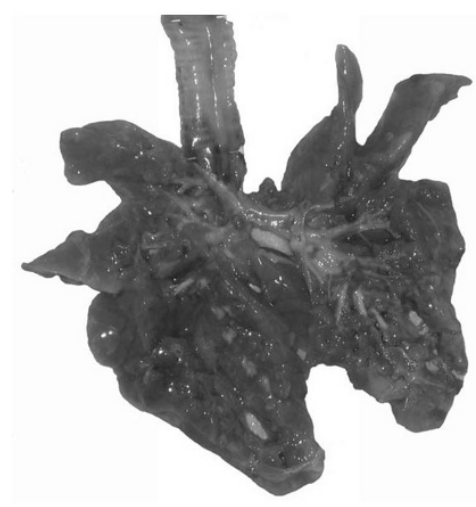

Figure 2. Dissected lung showing methylene blue stained surfactant administered via an LMA distributed throughout the bronchial tree.

videotape demonstrated only minimal leakage (estimated $0-0.5 \mathrm{~mL}$ ) of surfactant around the LMA inflatable cuff during surfactant administration. Dissection of the upper airway, lungs, and upper gastrointestinal system demonstrated staining of the lungs with only minimal staining of the esophagus and stomach (Fig. 2).

\section{Phase II}

Component 1: Placement of airway device. Videotape of the placement of the airway device was available for all pigs whereas HR and oxygen saturation data were not available for three pigs because of technical difficulties with the pulse oximeter. Time for successful placement of the LMA was $19 \pm 1 \mathrm{~s}$ versus $\mathrm{ETT} 123 \pm 35 \mathrm{~s}$ (mean $\pm \mathrm{SEM} ; p=0.01$ ). Number of attempts required for successful LMA placement was $1.1(1-2)$ versus ETT $1.9(1-7)$ [mean (range; $p=0.03)$ ]. Twenty-one of the 24 pigs $(88 \%)$ required only one attempt and three pigs required two attempts for successful placement of the LMA. This resulted in a total of 27 attempts with 96\% (26/27) of these attempts completed in $<30 \mathrm{~s}$. The remaining attempt required 33 s. ETT placement was successful on the first attempt in 16/24 $(67 \%)$ pigs. Four pigs $(17 \%)$ required two attempts, and three, five, six, and seven attempts were required for the remaining four pigs for a total of 45 attempts. Thirty-three percent (15/45) of these attempts were completed or terminated in $<30 \mathrm{~s}$. The average attempt for ETT placement was $64 \mathrm{~s}$.

Change in HR from baseline was not statistically different (LMA $1 \pm 14$ versus ETT $-3 \pm 17 \mathrm{bpm}, p=0.45$ ). Results for $\mathrm{SaO}_{2}$ levels during the procedure are presented in Table 1 . Duration of time with $\mathrm{SaO}_{2} \leq 85 \%$, lowest $\mathrm{SaO}_{2}$, and change in average $\mathrm{SaO}_{2}$ during the procedure compared with baseline showed statistical significance favoring the LMA group.

Component 2: Randomized, controlled trial. Thirty pigs were used for the randomized, controlled trial. Four pigs died or experienced severe lung injury (defined as persistent $\mathrm{PaO}_{2}<50$ $\mathrm{mm} \mathrm{Hg}$ ) during induction of lung injury, and appropriate lung injury was not able to be induced in two pigs, therefore 24 pigs ( $n=8$ /group) were analyzed (Fig. 3). Baseline characteristics did not differ between groups (Table 2). $\mathrm{PaO}_{2}$ levels in the LMA group were not statistically different compared with $\mathrm{PaO}_{2}$ levels in the ETT group. $\mathrm{PaO}_{2}$ levels in the LMA and ETT groups were significantly increased compared with controls $(p<0.05)$ (Fig.
Table 1. $\mathrm{SaO}_{2}$ results during placement of the airway device

\begin{tabular}{|c|c|c|c|}
\hline Variable & LMA & ETT & $p$ \\
\hline Baseline $\mathrm{SaO}_{2}(\%)$ & $89 \pm 12$ & $92 \pm 12$ & 0.48 \\
\hline $\mathrm{SaO}_{2} \leq 85 \%(\mathrm{~s})$ & $7 \pm 9$ & $47 \pm 80$ & $0.03 *$ \\
\hline $\mathrm{SaO}_{2} \leq 70 \%(\mathrm{~s})$ & $4 \pm 6$ & $24 \pm 65$ & 0.14 \\
\hline $\mathrm{SaO}_{2} \leq 60 \%$ (s) & $2 \pm 4$ & $15 \pm 46$ & 0.18 \\
\hline Lowest $\mathrm{SaO}_{2}(\%)$ & $79 \pm 22$ & $65 \pm 23$ & $0.02 *$ \\
\hline $\begin{array}{l}\text { Change in } \mathrm{SaO}_{2} \\
\text { from baseline }(\%) \dagger\end{array}$ & $-3 \pm 7$ & $-9 \pm 12$ & $0.04 *$ \\
\hline \multicolumn{4}{|c|}{$\begin{array}{l}\text { Values expressed as mean } \pm \mathrm{SD} \text {. } \\
* p<0.05 \text {. } \\
\dagger \mathrm{Calculated} \text { by subtracting the mean } \mathrm{SaO}_{2} \text { during the procedure from the } \\
\text { lean } \mathrm{SaO}_{2} \text { at baseline. }\end{array}$} \\
\hline
\end{tabular}

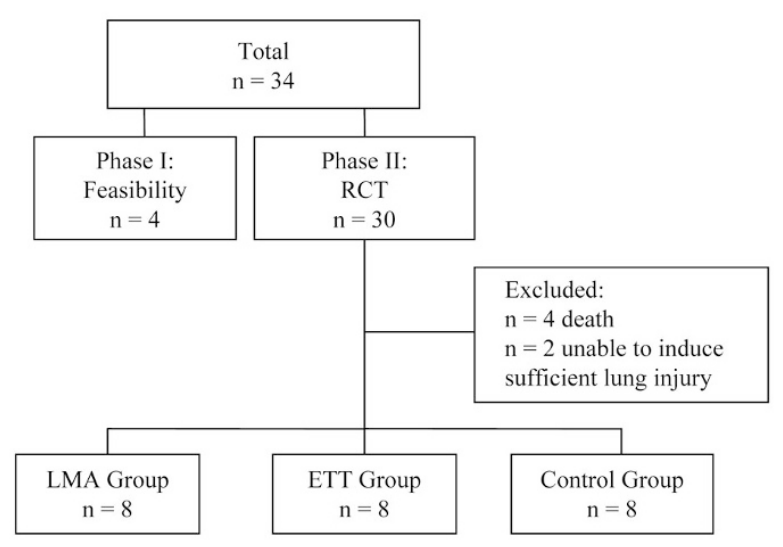

Figure 3. Trial profile.

4). $\mathrm{HR}, \mathrm{BP}$, and $\mathrm{pH}$ did not differ between groups. RR was lower in the ETT group compared with the LMA group and controls $(p<0.05)$ (Fig. 5).

\section{DISCUSSION}

In this two-part study, we investigated the use of a LMA compared with an ETT for administration of surfactant. Overall, we demonstrated that improvement in oxygenation in response to surfactant administered via an LMA did not differ from administration via an ETT and that the LMA device can be placed more easily and with less physiologic perturbations than an ETT.

During phase I of this study, we demonstrated the feasibility of using an LMA for surfactant administration in an animal model of respiratory distress syndrome. The pigs did not experience significant compromise with placement of the LMA or administration of surfactant. Real-time visualization and review of videotape of surfactant administration did not reveal significant leak around the LMA's inflated cuff. Visual examination of the postmortem lungs revealed staining throughout the lungs suggesting diffuse surfactant distribution. Minimal staining of the esophagus and stomach further supports the visualized findings of minimal leakage around the LMA cuff.

During phase II of this study, our results showed similar improvement in oxygenation in animals receiving surfactant via the LMA compared with those receiving endotracheal administration of surfactant. These data suggest that surfactant administered above the glottis via an LMA reaches the lungs and is distributed in a manner similar to surfactant that is administered 
Table 2. Baseline characteristics

\begin{tabular}{|c|c|c|c|c|c|c|c|}
\hline & \multirow[b]{2}{*}{ Weight (kg) } & \multicolumn{3}{|c|}{ Before lung injury } & \multicolumn{3}{|c|}{ Post lung injury } \\
\hline & & $\mathrm{pH}$ & $\mathrm{PaCO}_{2}$ & $\mathrm{PaO}_{2}$ & $\mathrm{pH}$ & $\mathrm{PaCO}_{2}$ & $\mathrm{PaO}_{2}$ \\
\hline Control $(n=8)$ & $1.59 \pm 0.20$ & $7.43 \pm 0.08$ & $37.50 \pm 6.48$ & $459.75 \pm 68.30$ & $7.43 \pm 0.10 *$ & $30.63 \pm 9.07$ & $132.63 \pm 21.47$ \\
\hline LMA $(n=8)$ & $1.73 \pm 0.27$ & $7.44 \pm 0.06$ & $37.88 \pm 6.88$ & $479.13 \pm 61.03$ & $7.33 \pm 0.05$ & $41.75 \pm 8.63$ & $123.75 \pm 26.29$ \\
\hline $\mathrm{ETT}(n=8)$ & $1.74 \pm 0.10$ & $7.41 \pm 0.06$ & $33.75 \pm 8.65$ & $426.38 \pm 55.86$ & $7.32 \pm 0.10 *$ & $40.25 \pm 12.49$ & $127.00 \pm 25.27$ \\
\hline
\end{tabular}

Values expressed as mean $\pm \mathrm{SD}$. Comparisons between groups were statistically nonsignificant except post lung injury pH, control vs ETT. $* p<0.05$.

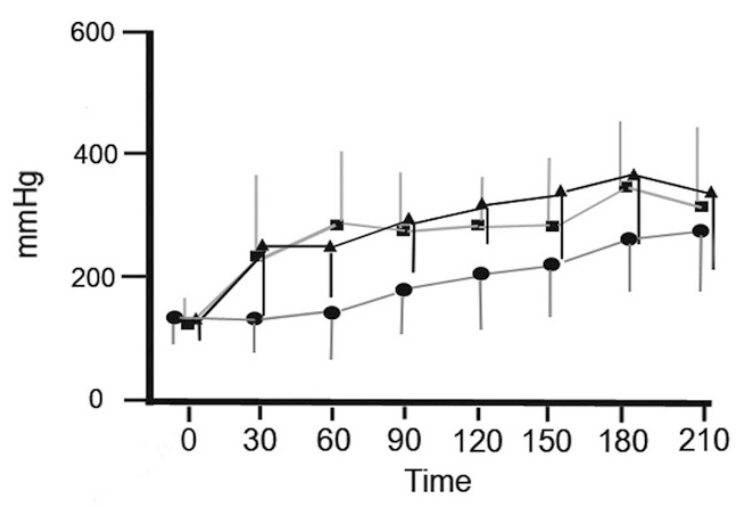

Figure 4. $\mathrm{PaO}_{2}$ levels in $\operatorname{LMA}(\square)$, ETT (A), and control (@) groups. Mean $\pm \mathrm{SD} ; p<0.05$, control vs LMA, ETT.

below the glottis via an ETT. In addition, the LMA was over six times faster and required only one-half the number of attempts to successfully place compared with the ETT.

The American Academy of Pediatrics Neonatal Resuscitation Program (NRP) recommends that an intubation attempt be terminated if the attempt exceeds $20 \mathrm{~s}$ (18). In a study investigating duration of intubation attempts, the authors found that $72 \%$ of intubation attempts were successful at $30 \mathrm{~s}$, whereas only $38 \%$ were successful at $20 \mathrm{~s}(p=0.001)$. Therefore, the authors concluded that a duration of $30 \mathrm{~s}$ is a reasonable guideline for neonatal intubation (19) and was the duration chosen for our study protocol. In our study, 96\% (26/27) of attempts to place a LMA were completed in $<30 \mathrm{~s}$, whereas only $33 \%(15 / 45)$ of the attempts to place an ETT were completed or terminated in $<30 \mathrm{~s}$. Despite our guidelines stating an attempt should be terminated at $30 \mathrm{~s}$, the average attempt for ETT placement was $64 \mathrm{~s}$. This reflects the difficulty in terminating an attempt, especially when vital signs are stable and/or the operator feels successful placement is eminent. Had the attempts which exceeded $30 \mathrm{~s}$ been terminated at $30 \mathrm{~s}$, our results for the average number of attempts required for successful ETT placement would have been higher.

Placing the LMA and administering surfactant through the LMA did not elicit negative side effects such as agitation or unfavorable changes in vital signs. Animals experienced $\mathrm{SaO}_{2}$ $\leq 85 \%$ for a shorter duration of time and the lowest $\mathrm{SaO}_{2}$ during the procedure was more favorable with placement of the LMA compared with an ETT. Laryngospasm secondary to administered of a liquid above the glottis is a potential complication of administering surfactant via an LMA. However, none of the pigs in our study showed signs of laryngospasm.

Developed in 1981, LMAs have been used in the adult and pediatric populations for over two decades. Although LMAs have been slow to make their appearance in the NICU setting, familiarity with the device is rapidly increasing. Previously published literature reports successful use of a LMA in neonates with difficult airways $(12,20)$, during routine neonatal resuscitation $(21,22)$, as a means of providing respiratory support for prolonged periods (23) and during neonatal transport (24-26).

To date, there have been two articles reporting use of LMAs for surfactant administration. The first is a case report of an LMA being used to administer surfactant to two infants (1.36 $\mathrm{kg}$ and $3.2 \mathrm{~kg}$ ) with $\operatorname{RDS}$ (13) whereas the second is a prospective study of eight preterm infants $(28-35 \mathrm{wk}, 880-$
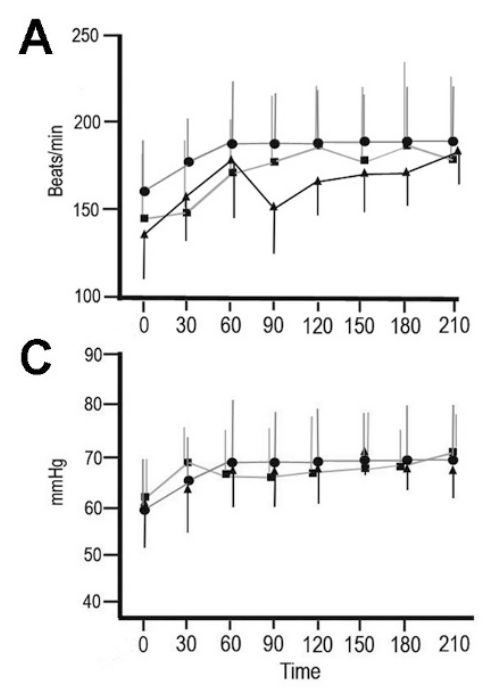

Figure 5. Physiologic and blood gas parameters in LMA $(\square)$, ETT $(\mathbf{\Delta})$, and control (○) groups. A. HR. B. RR. $p<$ 0.05 ETT vs LMA, control. C. BP. D. pH. All panels: mean $\pm \mathrm{SD}$. 
2520 g) with RDS (14). All infants were maintained on nCPAP before and after surfactant administration. In all cases, the arterial-alveolar oxygen tension ratio (a/A ratio) improved after surfactant administration without complication or hemodynamic changes.

Some limitations of our study deserve comment. Simulating human disease using an animal model creates challenges that may make extrapolation to humans difficult. However, an animal model provides useful and necessary information on the feasibility and safety of the device and protocol before experimentation in humans. Our study used pigs that have a laryngeal anatomy that differs from premature infants. The upper airway of a pig consists of blind pouches on either side of the glottis. This may result in a more difficult and prolonged intubation. To minimize bias because of this unique anatomy, intubations were performed by one of three providers who were familiar and highly skilled in pig intubation. In addition, pigs served as their own control by having an LMA placed followed by ETT placement and analyzing the results using a paired $t$ test. However, because the ETT was left in place for induction of acute lung injury, the LMA was always placed first. This may have resulted in the pig being more susceptible to increased agitation or changes in vital signs with placement of the ETT.

Newborn piglets with a weight of 1250 to $2500 \mathrm{~g}$ were used in this study to simulate the weight of preterm infants who may benefit from surfactant administration via an LMA. Methods for this animal trial, however, differ from a trial that would be conducted in human neonates. Although infants would remain supine after surfactant administration, the piglets were administered surfactant in the supine position and then suspended in the upright position, because this is their position of comfort and minimized agitation. This difference in positioning may affect the distribution of surfactant and should be considered when extrapolating results to infants who remain supine. The pigs also received continuous ketamine for sedation because this trial was conducted in accordance with the National Institutes of Health guidelines (15). Sedation with ketamine may affect the pig's response to airway device insertion or surfactant administration. Sedation in this animal model differs from human neonates where sedation and/or analgesia may vary or not be given at all.

Currently, infants with RDS require intubation with an ETT to receive surfactant. Although the size of the LMA limits its use in extremely premature infants, approximately $30 \%$ of infants $>1250$ g initially treated with nCPAP require subsequent intubation and mechanical ventilation (data obtained from the Vermont Oxford Network Nightingale Internet reporting system, a site with access restricted to members of the Network). If they were able to receive surfactant, some of these infants may be able to remain on nCPAP, thereby avoiding the potential adverse effects of intubation and mechanical ventilation. Although case reports suggest that LMAs are an effective means for administering surfactant, there are currently no published randomized controlled trials. By performing such a trial in an animal model of RDS and demonstrating that surfactant administration via an LMA is feasible and effective, we provide justification for a randomized, controlled trial in human neonates. If proven effective, some infants with respiratory distress may benefit from surfactant therapy while avoiding the associated risks of intubation and mechanical ventilation.

\section{REFERENCES}

1. Stevens TP, Blennow M, Soll RF 2004 Early surfactant administration with brief ventilation vs selective surfactant and continued mechanical ventilation for preterm infants with or at risk for respiratory distress syndrome. Cochrane Database Syst Rev 3:CD003063

2. Jobe AH 1993 Pulmonary surfactant therapy. N Engl J Med 328:861-868

3. Marshall TA, Deeder R, Pai S, Berkowitz GP, Austin TL 1984 Physiologic changes associated with endotracheal intubation in preterm infants. Crit Care Med 12:501-503

4. Kelly MA, Finer NN 1984 Nasotracheal intubation in the neonate: physiologic responses and effects of atropine and pancuronium. J Pediatr 105:303-309

5. Barrington KJ, Finer NN, Etches PC 1989 Succinylcholine and atropine for premedication of the newborn infant before nasotracheal intubation: a randomized, controlled trial. Crit Care Med 17:1293-1296

6. Millar C, Bissonnette B 1994 Awake intubation increases intracranial pressure without affecting cerebral blood flow velocity in infants. Can J Anaesth 41:281-287

7. Pokela ML, Koivisto M 1994 Physiological changes, plasma beta-endorphin and cortisol responses to tracheal intubation in neonates. Acta Paediatr 83:151-156

8. Friesen RH, Honda AT, Thieme RE 1987 Changes in anterior fontanel pressure in preterm neonates during tracheal intubation. Anesth Analg 66:874-878

9. Bhutada A, Sahni R, Rastogi S, Wung JT 2000 Randomized controlled trial of thiopental for intubation in neonates. Arch Dis Child Fetal Neonatal Ed 82:F34-F37

10. Kong AS, Brennan L, Bingham R, Morgan-Hughes J 1992 An audit of induction of anaesthesia in neonates and small infants using pulse oximetry. Anaesthesia 47:896-899

11. Raju TN, Vidyasagar D, Torres C, Grundy D, Bennett EJ 1980 Intracranial pressure during intubation and anesthesia in infants. J Pediatr 96:860-862

12. Berry AM, Brimacombe JR, Verghese C 1998 The laryngeal mask airway in emergency medicine, neonatal resuscitation, and intensive care medicine. Int Anesthesiol Clin 36:91-109

13. Brimacombe J, Gandini D, Keller C 2004 The laryngeal mask airway for administration of surfactant in two neonates with respiratory distress syndrome. Paediatr Anaesth 14:188-190

14. Trevisanuto D, Grazzina N, Ferrarese P, Micaglio M, Verghese C, Zanardo V 2005 Laryngeal mask airway used as a delivery conduit for the administration of surfactant to preterm infants with respiratory distress syndrome. Biol Neonate 87:217-220

15. National Research Council, Institute of Laboratory Animal Resources 2002 Guidelines for the care and use of laboratory animals (NIH 86-23). The National Academies Press, Washington, D.C

16. Lachmann B, Robertson B, Vogel J 1980 In vivo lung lavage as an experimental model of the respiratory distress syndrome. Acta Anaesthesiol Scand 24:231-236

17. Nold JL, Meyers PA, Worwa CT, Goertz RH, Huseby K, Schauer G, Mammel MC 2007 Decreased lung injury after surfactant in piglets treated with continuous positive airway pressure or synchronized intermittent mandatory ventilation. Neonatology 92:19-25

18. Kattwinkel J, ed 2006 Textbook of Neonatal Resuscitation, 5th ed. American Academy of Pediatrics and American Heart Association, Elk Grove Village, IL, pp 5-14

19. Lane B, Finer NN, Rich W 2004 Duration of intubation attempts during neonatal resuscitation. J Pediatr 145:67-70

20. Brimacombe J, Gandini D 1995 Resuscitation of neonates with the laryngeal mask airway-a caution. Pediatrics 95:453-454

21. Gandini D, Brimacombe JR 1999 Neonatal resuscitation with the laryngeal mask airway in normal and low birth weight infants. Anesth Analg 89:642-643

22. Trevisanuto D, Micaglio M, Pitton M, Magarotto M, Piva D, Zanardo V 2004 Laryngeal mask airway: is the management of neonates requiring positive pressure ventilation at birth changing? Resuscitation 62:151-157

23. Bucx MJ, Grolman W, Kruisinga FH, Lindeboom JA, Van Kempen AA 2003 The prolonged use of the laryngeal mask airway in a neonate with airway obstruction and Treacher Collins syndrome. Paediatr Anaesth 13:530-533

24. Trevisanuto D, Verghese C, Doglioni N, Ferrarese P, Zanardo V 2005 Laryngeal mask airway for the interhospital transport of neonates. Pediatrics 115:e109-e111

25. Brimacombe JR, Gandini D 1995 The laryngeal mask airway for helicopter transportation of neonates. Med J Aust 162:56

26. Fraser J, Hill C, McDonald D, Jones C, Petros A 1999 The use of the laryngeal mask airway for inter-hospital transport of infants with type 3 laryngotracheo-oesophageal clefts. Intensive Care Med 25:714-716 